\title{
Knotted and linked phase singularities in monochromatic waves
}

\author{
By M. V. BERRY AND M. R. DENNIS \\ H. H. Wills Physics Laboratory, Tyndall Avenue, Bristol BS8 1TL, UK \\ Received 22 November 2000; accepted 29 March 2001
}

Exact solutions of the Helmholtz equation are constructed, possessing wavefront dislocation lines (phase singularities) in the form of knots or links where the wave function vanishes ('knotted nothings'). The construction proceeds by making a nongeneric structure with a strength $n$ dislocation loop threaded by a strength $m$ dislocation line, and then perturbing this. In the resulting unfolded (stable) structure, the dislocation loop becomes an $(m, n)$ torus knot if $m$ and $n$ are coprime, and $N$ linked rings or knots if $m$ and $n$ have a common factor $N$; the loop or rings are threaded by an $m$-stranded helix. In our explicit implementation, the wave is a superposition of Bessel beams, accessible to experiment. Paraxially, the construction fails.

Keywords: phase; singularities; dislocations; knots; links; paraxiality

\section{Introduction}

Wavefront dislocations, that is, phase singularities in complex scalar waves (Nye \& Berry 1974; Berry 1981; Nye 1999) (also known as optical vortices), are lines in three dimensions on which the wave intensity vanishes and around which the phase changes by $2 \pi$ times an integer (the strength of the singularity). For any wave in space, the set of its dislocation lines is a skeleton, supporting the phase structure of the whole field.

It is already known that solutions of the wave equation (monochromatic or time dependent) can be constructed to represent dislocation lines that are straight or curved, or form closed loops. In view of the recent revival of interest in these singular structures (Soskin 1997; Vasnetsov \& Staliunas 1999), it is desirable to explore all their possible geometries. Our purpose here is to address the natural question: can wavefront dislocations be knotted or linked?

In reaction-diffusion equations describing chemical waves, wave functions with knotted dislocations have been created as initial conditions, and their structure and evolution have been studied in detail (Winfree 1987; Winfree \& Strogatz 1984; Winfree et al. 1985; T. Poston \& A. T. Winfree 1992, unpublished work). We impose the more demanding condition that the knots remain stationary. Specifically, we seek knots and links in complex scalar wave solutions $\Psi(\boldsymbol{r})$ of the Helmholtz equation

$$
\nabla^{2} \Psi(\boldsymbol{r})+\Psi(\boldsymbol{r})=0
$$

(We measure distances in units of wavelength $/ 2 \pi$, or, equivalently, choose the wave number $k=1$.) For $\boldsymbol{r}$, we will use cylindrical polar coordinates $\boldsymbol{r}=(R, \phi, z)$ or Cartesians $\boldsymbol{r}=(x, y, z)$. 


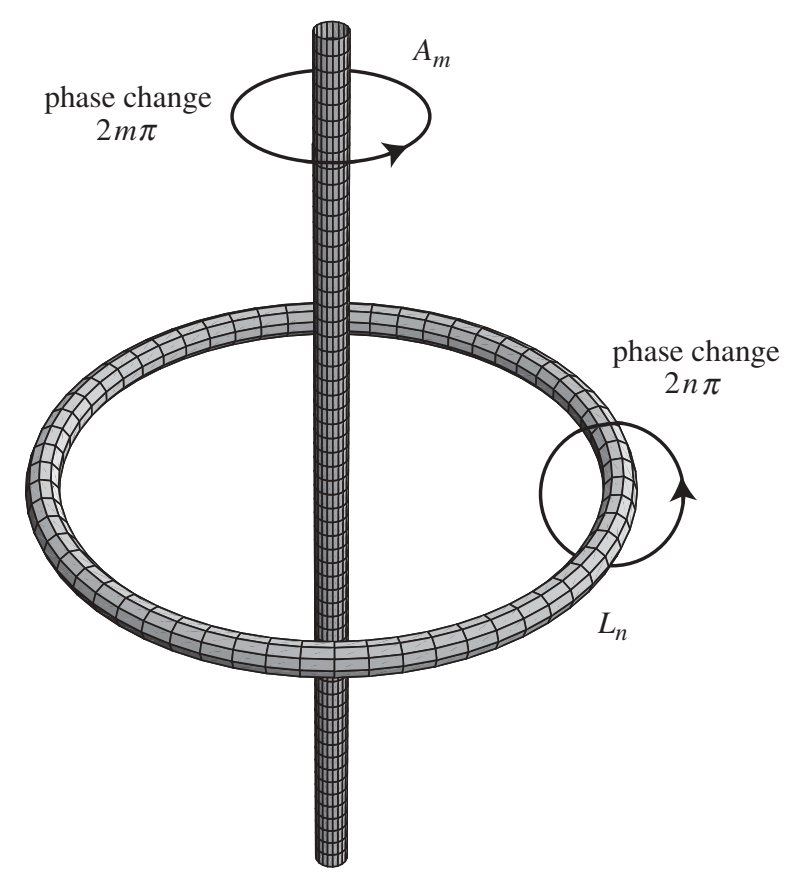

Figure 1. Unstable strength $m$ axial dislocation $A_{m}$ threading an unstable strength $n$ dislocation loop $L_{n}$.

We answer our question in the affirmative, by constructing explicit exact solutions of (1.1) containing dislocation knots and links (zeros of $\Psi$ ), using a combination of topology and analysis. In the basic strategy $(\S 2)$, we envisage the knot or link collapsed into a structurally unstable high-order dislocation ring, threaded by an axial high-order dislocation, and then argue that under perturbation the ring will unfold into the desired knot or link. To implement this strategy requires explicit solutions with definite properties, and we choose, from many possible types of solution, a particularly convenient set $(\S 3)$ consisting of superpositions of Bessel beams.

A surprising aspect of the analysis is that although it works for the Helmholtz equation (1.1), it fails for the paraxial approximation to (1.1). This is a consequence of the result (Appendix D), which is of independent interest, that paraxial wave equations cannot possess solutions with dislocation loops whose strength exceeds unity. Two other results arising from this work, which are also of independent interest, are a clarification (Appendix A) of the local structure of higher-order dislocations, and a topological theorem (Appendix B) showing that screw dislocation loops must be threaded by other dislocations.

\section{Unstable dislocation structures and their unfoldings}

The starting point of our construction will be an unstable (non-generic) structure consisting of a circular dislocation loop $L_{n}$ of strength $n>0$, lying in the plane $z=0$ and with radius $R^{*}$, centrally threaded by a strength $m$ dislocation line $A_{m}$ along the $z$-axis (figure 1). Then we will perturb this to get the desired knot or link as the stable unfolding of $L_{n}$. To create the unstable structure, we first note that near $A_{m}$ 
the local field must be

$$
K R^{m} \exp (\mathrm{i} m \phi),
$$

where $K$ is a constant. In Appendix A we show that, near $L_{n}$, the local field must be

$$
\left(K_{+}\left(R-R^{*}+\mathrm{i} z\right)^{n}+K_{-}\left(R-R^{*}-\mathrm{i} z\right)^{n}\right) \exp (\mathrm{i} m \phi),
$$

where $K_{ \pm}$are constants with $\left|K_{+}\right|>\left|K_{-}\right|$.

Thus we write the unperturbed wave in the form

$$
\psi(\boldsymbol{r})=\exp (\mathrm{im} \phi) F(R, z)
$$

and seek to create the desired structure by imposing conditions on $F$. For $A_{m}$, we require, from (2.1),

$$
\left.\begin{array}{l}
\partial_{R}^{p} \psi(0, z)=0, \quad 0 \leqslant p \leqslant m-1, \\
\partial_{R}^{m} \psi(0, z) \neq 0,
\end{array}\right\}
$$

where, here and hereafter, we denote derivatives with respect to a variable $\xi$ by the symbol $\partial_{\xi}$. For the loop $L_{n}$, we require, from $(2.2)$, the $\frac{1}{2} n(n+1)$ conditions

$$
\left.\begin{array}{ll}
\partial_{R}^{q} \partial_{z}^{p-q} \psi\left(R^{*}, 0\right)=0, & 0 \leqslant q \leqslant p, \quad 0 \leqslant p \leqslant n-1, \\
\partial_{R}^{q} \partial_{z}^{n-q} \psi\left(R^{*}, 0\right) \neq 0, & 0 \leqslant q \leqslant n .
\end{array}\right\}
$$

In effect, we are envisaging (imagining the unfolding in reverse) that $n$ strength 1 dislocations coalesce into a single strength $n$ dislocation. This requires $\frac{1}{2} n(n+1)$ conditions, rather than just $n-1$; the greater number arises because a strength $n$ dislocation has Poincaré index +1 for any $n$, whose conservation (in addition to dislocation strength) during the coalescence implies the simultaneous involvement of $n-1$ phase saddles (each with index -1) (Nye et al. 1988; Berry 1998).

Around any circuit threaded by $L_{n}$, the phase $\chi$ changes by $2 \pi n$. Alternatively stated, for each value of $\chi(\bmod 2 \pi)$, there are $n$ wavefront surfaces emerging from $L_{n}$; any transverse section of $L_{n}$ cuts these surfaces in $n$ lines, issuing from the intersection point, comprising what we call the phase star (parts $(a)-(c)$ of figure 2 ). Around any circuit threaded by $A_{m}$, the phase changes by $2 \pi m$. By taking such a circuit to be a loop just inside $L_{m}$, we can interpret this as a constraint on the phase star: along $L_{n}$, the phase star must turn $m / n$ times. This implies a helicoidal structure in the wavefronts issuing from $L_{n}$, and illustrates a general theorem (Appendix B): the phase change along a dislocation loop equals the total strength of the dislocations threading it.

Now let this $A_{m}, L_{n}$ structure be perturbed by an additional weak wave $\psi_{p}(\boldsymbol{r})$ that does not itself possess any dislocation lines threading $L_{n}$ (for example, $\psi_{p}$ could be a plane wave). Thus the total wave is

$$
\Psi(\boldsymbol{r})=\psi(\boldsymbol{r})+\varepsilon \psi_{p}(\boldsymbol{r})=\exp (\mathrm{im} \phi) F(R, z)+\varepsilon \psi_{p}(\boldsymbol{r}) .
$$

This splits $L_{n}$ into $n$ separate dislocation strands with strength 1 (zeros of $\Psi$ ), and in each cross-section the phase star splits into $n$ strength 1 stars (parts $(d)-(f)$ of figure 2) and $n-1$ phase saddles (if the unfolding is incomplete, the saddles can be degenerate, as in figure $2 e$ ).

If $m$ and $n$ are coprime, these strands cannot take the form of $n$ separate dislocation loops, because each of the $n$ phase stars will be unable to match smoothly with its 

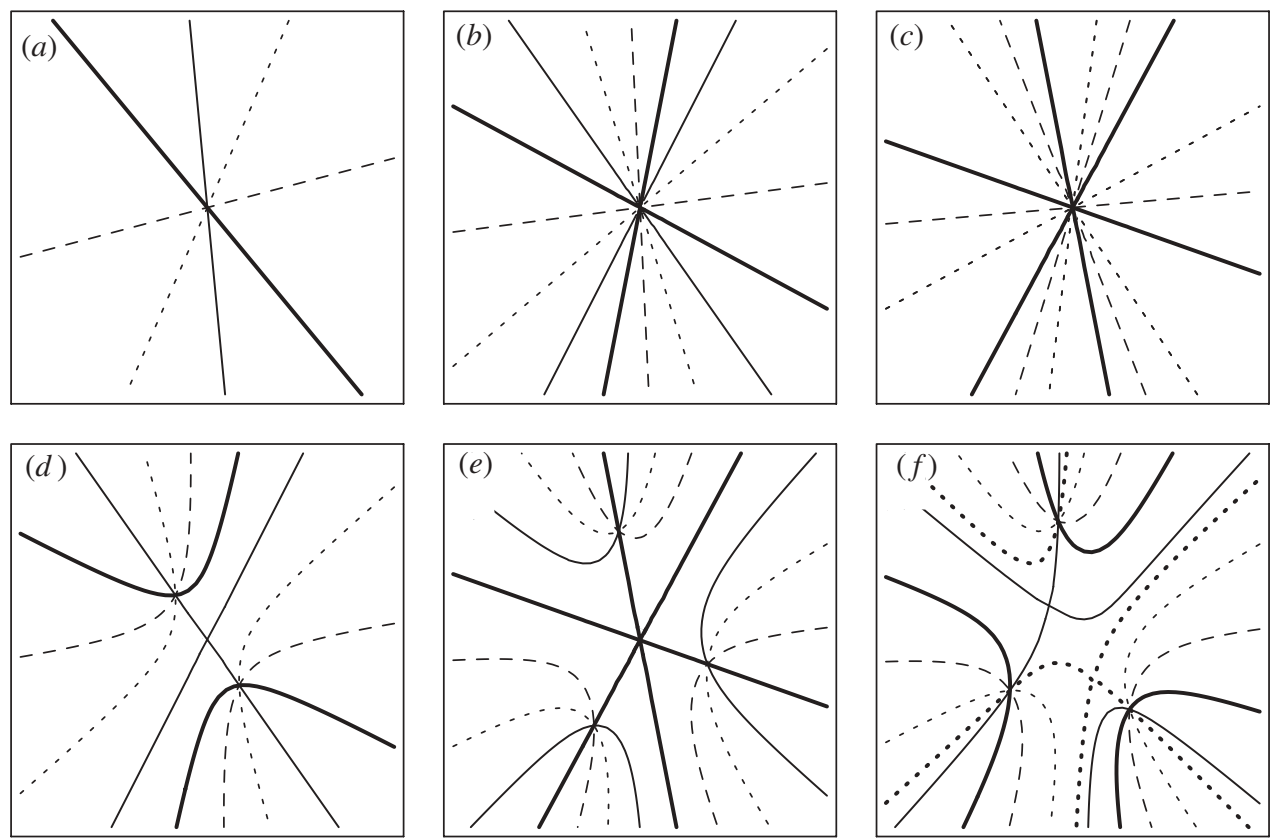

Figure 2. Phase stars. Thick lines: $\chi=0$ and $\pi(\bmod 2 \pi)($ crests and troughs of $\operatorname{Re} \psi)$; dashed lines: $\chi=\frac{1}{8} \pi$ and $\frac{5}{8} \pi(\bmod 2 \pi)$; thin lines: $\chi=\frac{1}{2} \pi$ and $\frac{3}{2} \pi(\bmod 2 \pi)$; light dotted lines: $\chi=\frac{3}{8} \pi$ and $\frac{7}{8} \pi(\bmod 2 \pi)$; heavy dotted lines in $(f): \chi=2.671 \pi / 8$. (a) Strength 1 dislocation $L_{1}$; (b) unstable strength 2 dislocation $L_{2} ;(c)$ unstable strength 3 dislocation $L_{3} ;(d)$ stable unfolding of $(b) ;(e)$ partial unfolding of $(c) ;(f)$ stable unfolding of $(c)$.
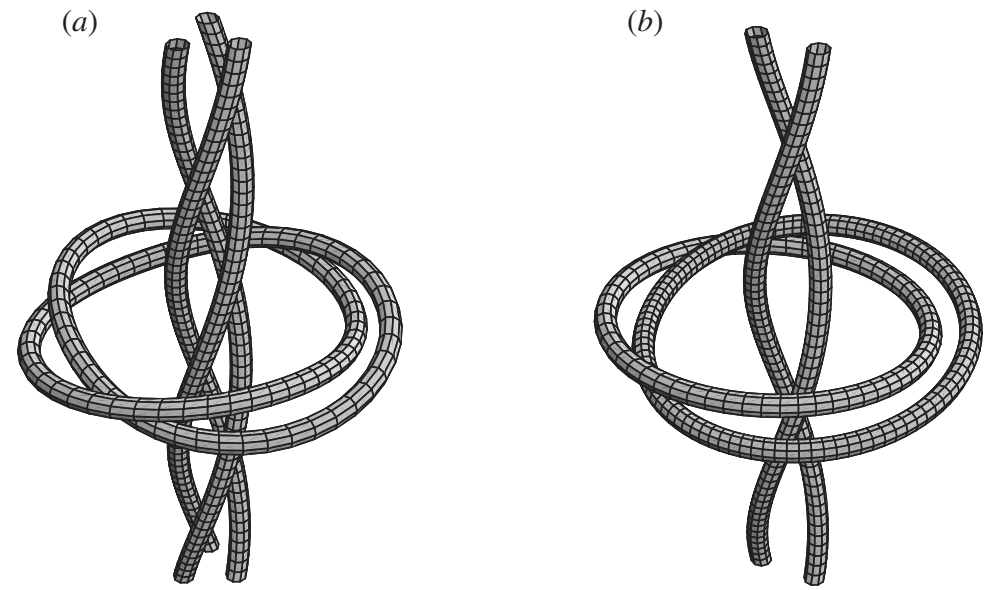

Figure 3. Stable unfoldings of figure 1. (a) The $(m=3, n=2)$ (trefoil) knot threaded by a triple-stranded helix. (b) The $(m=2, n=2)$ link, threaded by a double-stranded helix.

beginning after turning $m / n$ times in a circuit of the axial dislocation. Thus the strands form a single loop that turns $n$ times round the $z$-axis before closing. Now, we show in Appendix $\mathrm{C}$ that the cluster of $n$ unfolded phase stars must be convected by the phase pattern that it carries. It follows that the $n$ strands must also twist $m$ times round the original loop $L_{n}$ before closing. Therefore, the unfolding of $L_{n}$ is an 
$(m, n)$ torus knot (Adams 1994). Similarly, $A_{m}$ must unfold into $m$ separate strength 1 dislocation lines, and we will soon see that they form an $m$-stranded helix. The fully unfolded structure, of an $(m, n)$ torus knot threaded by an $m$-stranded helix, is illustrated for the trefoil in figure $3 a$.

If $m$ and $n$ are not coprime, but share a common multiple, so that $(m, n)=$ $N\left(m_{0}, n_{0}\right)$, with $m_{0}, n_{0}$ coprime, the 'knot' into which $L_{n}$ unfolds consists of $N$ identical linked loops, each of which is an $\left(m_{0}, n_{0}\right)$ knot, and the whole set of $N$ loops is threaded by an $m$-stranded helix. Figure $3 b$ illustrates this for the simplest link. It is possible for the individual linked dislocations to be knotted; the simplest situation where this occurs is $m=6, n=4$, corresponding to two linked trefoil knots.

These topological arguments can be confirmed and extended by explicit calculations of the zeros of (2.6) for small $\varepsilon$. It will suffice to consider perturbations $\psi_{p}$ with circular symmetry, that is, $\psi_{p}$ depends only on $R$ and $z$. Consider first the neighbourhood of $L_{n}$. Using the local form (2.2) for the unperturbed wave, and defining the constant

$$
B_{L} \equiv \psi_{p} \quad\left(\boldsymbol{r} \text { on } L_{n}\right),
$$

we have, as the equation for the unfolding of $L_{n}$,

$$
K_{+} \rho^{n} \exp (\mathrm{i} n \gamma)+K_{-} \rho^{n} \exp (-\mathrm{i} n \gamma)=-\varepsilon B_{L} \exp (-\mathrm{i} n \phi),
$$

where $\rho, \gamma$ are polar coordinates in azimuthal sections of $L_{n}$ defined by

$$
R-R^{*}+\mathrm{i} z=\rho \exp (\mathrm{i} \gamma) .
$$

According to (2.8), and remembering that $\left|K_{+}\right|>\left|K_{-}\right|, n \gamma$ increases by $2 \pi$ as $m \phi$ decreases by $2 \pi$. It follows that along each strand ( $\phi$ changing by $2 \pi), \gamma$ changes by $2 \pi m / n$, so along the whole knot ( $\phi$ changing by $2 \pi n), \gamma$ changes by $2 \pi m$. Thus the knot is indeed an $(m, n)$ torus knot if $m$ and $n$ are coprime, and a link otherwise. On each azimuthal section specified by $\phi$, the $n$ solutions $\gamma$ (of (2.8)), corresponding to the different strands, lie on a circle with radius $\rho=O\left(\varepsilon^{1 / n}\right)$; the union of all these circles is the torus, with coordinates $\gamma$ and $\phi$, on which the dislocation knot is wound.

Consider now the neighbourhood of $A_{m}$, and define the function

$$
B_{A}(z)=\psi_{p} \quad\left(\boldsymbol{r} \text { on } A_{m}\right) .
$$

Then an analogous argument based on (2.1) shows that the $m$ strength 1 dislocations into which $A_{m}$ unfolds must lie on the surface

$$
R(z)=\varepsilon^{1 / m}\left|B_{A}(z) / K\right|^{1 / m} .
$$

This is a tube whose cross-section, with radius of order $\varepsilon^{1 / m}$, varies with $z$. For the $m$ strands, we obtain

$$
\phi_{j}(z)=\frac{\arg \left(-B_{A}(z) / K\right)}{m}+\frac{2 \pi j}{m}, \quad 1 \leqslant j \leqslant m .
$$

Since $B_{A}(z)$, in fact, must vary with $z$ (if $\psi_{p}$ satisfies the wave equation and has circular symmetry), the strands rotate and are therefore braided into an $m$-stranded helix, as claimed. (The sense in which the helix twists with $z$ is unrelated to the sense in which the strands of the knot twist with $\phi$.) 


\section{Bessel knots}

At first thought, it would seem simplest-both for theoretical calculation and for experimental realization - to implement the above procedure with the LaguerreGauss beams of paraxial optics (Allen et al. 1992). However, this attempt fails, because conditions (2.5) for the circular dislocation $L_{n}$ are impossible to satisfy with any paraxial waves. This follows from the general result, proved in Appendix D, that there can be no higher-order dislocation loops in solutions of the paraxial wave equation.

From the many classes of exact solutions of (1.1) in the form (2.3), we choose the $m$-Bessel beams (Durnin 1987; Durnin et al. 1987)

$$
F_{m b}(R, z)=J_{m}(b R) \exp \left(\mathrm{i} z \sqrt{1-b^{2}}\right) .
$$

Here, $b$ is a constant and $0 \leqslant b \leqslant 1$ for the non-evanescent waves we are interested in. Such beams automatically satisfy (2.4) and so possess the desired axial dislocation $A_{m}$. To satisfy (2.5), we choose real constants $b_{1}, \ldots, b_{n(n+1) / 2}$ and construct the superposition

$$
F(R, z)=\sum_{l=1}^{n(n+1) / 2} a_{l} F_{m b_{l}}(R, z),
$$

with real constants $a_{l}$. For simplicity, we can choose $b_{1}=1$ and, without loss of generality, we can take $a_{1}=1$. Then, for a fixed choice of the remaining $b_{l}$, we can adjust the remaining $a_{l}$ and $R^{*}$ to ensure that (2.5) is satisfied, thereby making $n$ zeros of the Bessel superposition coalesce, creating the desired loop $L_{n}$.

As examples, we now create a $(3,2)$ trefoil knot and a $(2,2)$ link. In both cases, construction of the loop $L_{2}$ involves three Bessel functions (of order 3 for the knot, 2 for the link), for which we choose the scaling factors

$$
b_{1}=1, \quad b_{2}=\frac{1}{3}, \quad b_{3}=\frac{2}{3} .
$$

The coefficients $a_{2}, a_{3}$ (with $a_{1}=1$ ), and the radius $R^{*}$, are determined by the equations (2.5), which for the knot become, incorporating (3.1),

$$
\left.\begin{array}{rl}
F\left(R^{*}, 0\right) & =J_{3}\left(R^{*}\right)+a_{2} J_{3}\left(\frac{1}{3} R^{*}\right)+a_{3} J_{3}\left(\frac{2}{3} R^{*}\right)=0, \\
\partial_{R} F\left(R^{*}, 0\right) & =J_{3}^{\prime}\left(R^{*}\right)+\frac{1}{3} a_{2} J_{3}^{\prime}\left(\frac{1}{3} R^{*}\right)+\frac{2}{3} a_{3} J_{3}^{\prime}\left(\frac{2}{3} R^{*}\right)=0, \\
\partial_{z} F\left(R^{*}, 0\right) & =\frac{1}{3} \sqrt{8} a_{2} J_{3}\left(\frac{1}{3} R^{*}\right)+\frac{1}{3} \sqrt{5} a_{3} J_{3}\left(\frac{2}{3} R^{*}\right)=0
\end{array}\right\}
$$

(for the link, the equations are the same, but with the Bessel indices 3 replaced by 2). These equations are easy to solve numerically. Indeed, there are many solutions, and we choose those corresponding to coalescence of the two smallest zeros of the Bessel superpositions. The results are

$$
\left.\begin{array}{llll}
a_{2}=10.0302, & a_{3}=-3.18960, & R^{*}=5.44992 & ((3,2) \text { trefoil knot }), \\
a_{2}=4.73341, & a_{3}=-2.70176, & R^{*}=4.32636 & ((2,2) \text { link })
\end{array}\right\}
$$

and constitute the data needed to construct the loops $L_{2}$.

As the perturbation that converts the unstable $L_{2}$ into the stable knot or link, we take the zero-Bessel beam

$$
\psi_{p}(\boldsymbol{r})=J_{0}\left(\frac{1}{4} R\right) \exp \left(\frac{1}{4} \mathrm{i} \sqrt{15} z\right) .
$$


(a)

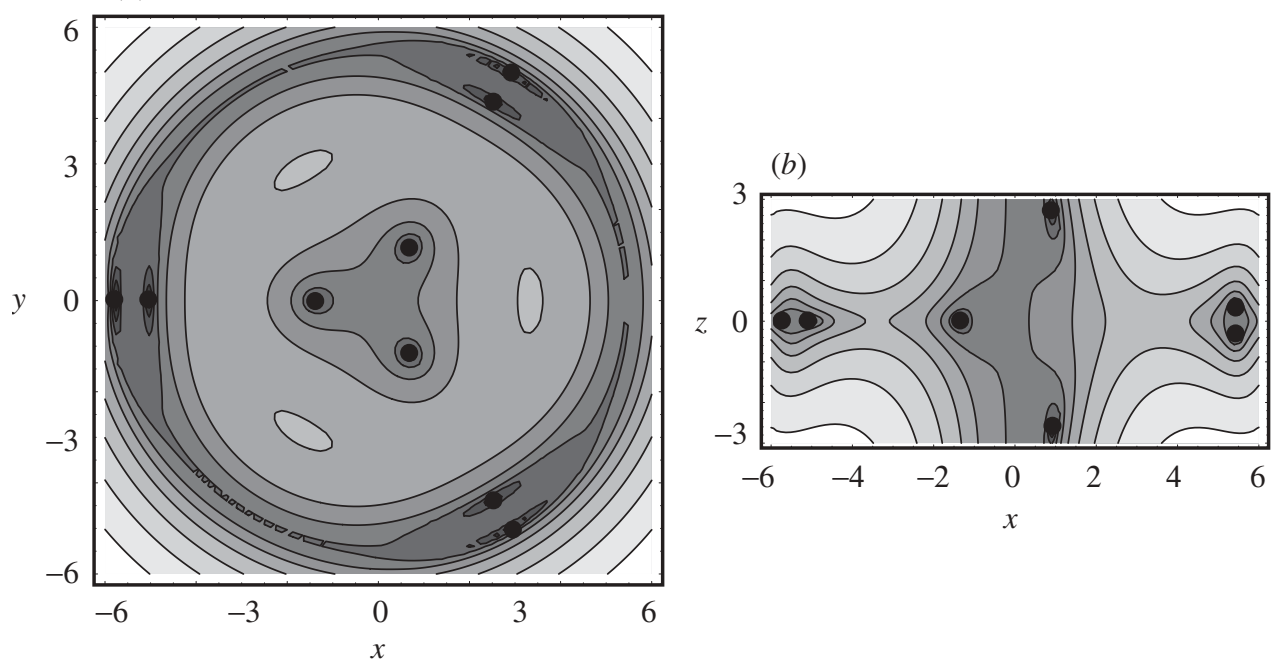

(c)
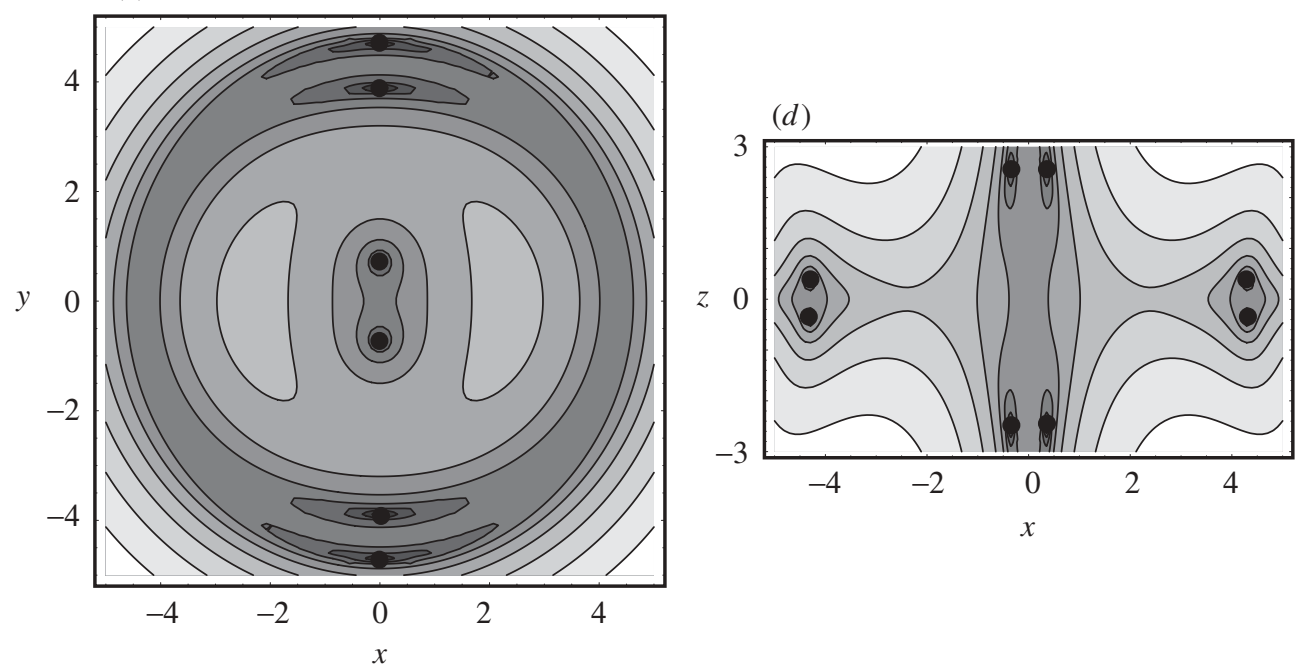

Figure 4. Density plots of wave intensity for the superposition (2.6), (3.2) and (3.5), with $\psi_{p}$ given by (3.6) and $\varepsilon=0.02$, in the planes $(a)(c) z=0$ and $(b),(d) x=0$, for the $(a),(b)(m=3, n=2)$ trefoil knot and the $(c),(d)(m=2, n=2)$ link. Black dots indicate the points where dislocation lines intersect these planes. The dislocation knot is threaded by a triple-stranded dislocation helix, and the dislocation link by a double-stranded dislocation helix.

Figure 4 shows sections of the total (that is, perturbed) waves for the two cases, one with its dislocation knotted, the other linked.

\section{Concluding remarks}

By constructing an unstable dislocation structure with high symmetry and then perturbing it, we are able to create waves with a dislocation in the form of any knot or link that can be wound round a torus. For the explicit construction, we chose the 
superposition (3.2) of Bessel beams (3.1). However, there are many alternative sets of exact solutions of (1.1), of the form (2.3) with definite angular momentum, that have sufficient flexibility to satisfy conditions (2.5). For example, we can take 'polynomial waves' (Nye 1998; Berry 1998), where $F(R, z)=\exp (\mathrm{i} z) \times($ polynomial in $R, z)$, and the calculations are almost identical to those in $\S 3$.

Optical fields satisfy the Helmholtz equation in the scalar approximation, raising the possibility that the spatially fixed and geometrically stable dislocation knots and links that we have created theoretically could be manufactured experimentally. Bessel beams are good candidates for such realization, since they can be manipulated experimentally (Durnin et al. 1987). But the essentially non-paraxial nature of the construction (see below and Appendix D) presents a challenge to experimentalists, especially since the scalar approximation is problematic for non-paraxial light (of course, this particular difficulty would not arise for longitudinal fields such as sound in a fluid).

It was surprising to discover (Appendix D) that paraxial approximations to the wave equation are inadequate to implement our strategy for creating knots. We cannot exclude the possibility that paraxial fields can support knots generated by other means, but there would be a prohibition against collapsing the $n$ strands of such a paraxial knot into a single strength $n$ dislocation loop. The prohibition could originate, for example, in an extraneous dislocation passing between the strands; we see no way to construct such an exotic object.

The non-existence of paraxial loops with strength greater than unity is interesting in its own right, and for at least two reasons. First, it is unusual for a wave equation to restrict the possible morphologies of phase singularities.

Second, non-paraxial beams possess axial dislocation rings of strength unity and all of the same sign (Berry 1994), which can be mimicked paraxially (Karman et al. 1997, 1998) by diffraction of a Gaussian beam by an aperture. In both paraxial and nonparaxial cases, these rings can be manipulated by varying a parameter, to undergo a series of singular events (for example, a +1 dislocation loop colliding with a loop of saddles and exploding into a strength -1 loop flanked by two +1 loops). However, one 'supersingular' event that can occur non-paraxially (Nye 1998; Berry 1998), but that is paraxially impossible, is the (two-parameter) steering of loops of strength unity to coincide pairwise into loops of strength 2. Detailed examination of the algebra describing the supersingular event shows that in the equivalent paraxial situation there is always an additional extraneous loop, of opposite strength to the two that are being steered into coincidence, which arrives simultaneously and prevents the formation of the strength 2 loop.

Phase singularities in three dimensions are vortex lines in the current $\boldsymbol{j}=\operatorname{Im} \psi^{*} \nabla \psi$, so our constructions are vortex knots, as studied in hydrodynamics (Moffatt 1969; Moffatt \& Ricca 1992). These waves could describe stationary configurations of electrons in free space (because in this situation the Schrödinger equation reduces to the Helmholtz equation); similar solutions exist in the presence of the Coulomb field from an atomic nucleus (Berry 2001), and these knotted vortices can describe states of electrons in atoms, perhaps as envisaged by Kelvin $(1867,1869)$.

Although the $(m, n)$ construction includes a wide variety of knots and links, there are many knots that cannot be smoothly wound on a torus, and so the strategy described here does not generate them. The obvious question arises whether every topology of dislocation can be sculpted in a monochromatic wave. For example, is it 
possible to make the Borromean rings (Cromwell et al. 1998), where three loops are entangled but no two are linked?

Dislocation knots can occur, but are they typical? For example, in the sound waves in a room filled with conversation the air contains a forest of dislocation linesthreads of silence - and it is reasonable to suppose that some of the dislocations are knotted. But how many? A precise formulation is as follows. In a Gaussian random wave field with prescribed power spectrum, what fraction of the points where dislocations pierce any two-dimensional section corresponds to dislocation lines that are part of a knot? This is a difficult problem in statistical geometry, but techniques exist (Berry \& Dennis 2000) that may be adaptable to deal with topological questions.

M.V.B.'s research is supported by The Royal Society; in addition, he is grateful for the hospitality of the Technion, Haifa, where the first draft of this paper was written. M.R.D. is supported by a University of Bristol postgraduate scholarship. We thank Professor Arthur Winfree for helpful discussions, and for sending us his unpublished paper with Professor Timothy Poston.

\section{Note added in proof}

As a result of computations kindly communicated to us by Professor Miles Padgett and Dr Johannes Courtial, we now appreciate that the paraxial prohibition against higher-order dislocation loops does not prevent the formation of knots in paraxial waves. In terms of our strategy, it suffices to apply a 'perturbation' to a wave with $n$ neighbouring parallel dislocation loops of strength 1, rather than a single loop of strength $n$, provided the 'perturbation' is large enough. Such $n$-loop complexes can be created easily by replacing the set of radial wavenumbers $b_{l}$ by very small, even paraxial, values with the same ratios, but using the same parameters $a_{l}$ that we have specified (e.g. in (3.5)).

\section{Appendix A. Local structure of higher-order Helmholtz dislocations}

Let the strength $n$ dislocation pass through the plane $y=0$ in the positive $y$ direction, and choose polar coordinates $\rho, \gamma$ (cf. (2.9)) in the $x, z$-plane. Therefore, the phase of $\psi$ must increase by $2 \pi n$ as $\gamma$ increases by $2 \pi$. It follows that the Fourier expansion of $\psi$ begins with the terms $\exp ( \pm \mathrm{i} n \gamma)$, and smoothness demands that $\psi$ vanishes as $\rho^{n}$ on the dislocation. This, in turn, implies that $\partial_{x}^{2} \psi$ and $\partial_{z}^{2} \psi$ vanish as $\rho^{n-2}$, and so dominate the term $\psi$ in the Helmholtz equation (1.1); they also dominate the term $\partial_{y}^{2} \psi$ in (1.1), because the derivative along the dislocation vanishes to higher order (i.e. $n$ ). Thus, close to the dislocation, the transverse dependence of $\psi$ must satisfy Laplace's equation

$$
\partial_{x}^{2} \psi+\partial_{z}^{2} \psi=0
$$

The most general solution proportional to $\rho^{n}$ is

$$
\psi=K_{+}(x+\mathrm{i} z)^{n}+K_{-}(x-\mathrm{i} z)^{n}=\rho^{n}\left[K_{+} \exp (\mathrm{i} n \gamma)+K_{-} \exp (-\mathrm{i} n \gamma)\right] .
$$

Around the dislocation, that is, as $\gamma$ increases by $2 \pi$, the term with coefficient $K_{+}$ winds $n$ times positively in the complex plane of $\psi$, and the term with $K_{-}$winds negatively. For $\psi$ to represent a strength $+n$ dislocation, the term $K_{+}$must dominate, that is, $\left|K_{+}\right|>\left|K_{-}\right|$(otherwise, $\psi$ represents a strength $-n$ dislocation). Translating 
to coordinates near the circular dislocation created in $\S 2$, and incorporating the required azimuthal dependence, we obtain (2.2).

The local behaviour (A 2) is a strong restriction on $\psi$, imposed by the Helmholtz equation. It implies that, around a circle centred on the dislocation, the wave $\psi$ describes an ellipse in the complex $\psi$-plane, repeated $n$ times, whose eccentricity depends on $K_{+} / K_{-}$. Without the Helmholtz requirement, $\psi$ would be a superposition of monomials of the form $x^{j} z^{n-j}$, whose variation in its complex plane is more complicated than an ellipse.

\section{Appendix B. Screw dislocation loops must be threaded (see also Winfree (1987) and Winfree et al. (1985))}

Recall that the strength $S$ of a dislocation is defined as the number of cycles of phase $\chi$ in a circuit $C$ around it,

$$
2 \pi S=\oint_{C} \mathrm{~d} \chi
$$

This defines a direction along the singular line, namely that which is right handed with respect to $C$ when $S>0$.

Imagine now an unknotted dislocation loop $L$ of strength 1 . Along $L$, the phase star (figure 2a) usually rotates (that is, it has screw character (Nye \& Berry 1974)), and by continuity, the number of rotations must be a signed integer; this is the screw number $m$. For convenience, we define $m>0(<0)$ if the screw is left (right) handed with respect to the dislocation direction. If the wavefront surface $\chi=$ const. $(\bmod 2 \pi)$ near $L$ is regarded as a ribbon, then the integer $m$ is the linking number of its edges.

On a closed curve $C$ just inside $L$ (so that there are no dislocations between $C$ and $L$ ), the phase cycles $m$ times as the star rotates, that is,

$$
2 \pi m=\oint_{C} \mathrm{~d} \chi
$$

showing that the screw number $m$ must be equal to the dislocation strength threading $C$ and also $L$.

If the dislocation strength of $L$ is $n>1$, so that its phase star is multiple (parts (b) and $(c)$ of figure 2 ), then the screw number (still defined as the number of rotations) is quantized in units of $1 / n$, and the dislocation strength threading the loop is equal to the number of such $1 / n$ rotations of the multiple phase star about $L$. If the screw number is $m / n$, then $m$ is the linking number of the wavefront ribbon of the torus knot produced by the perturbation described in $\S 2$.

If the threading dislocation $A$ is itself a loop (that is, if $A$ and $L$ are linked), then it has a screw number equal to the strength $n$ of $L$ : each loop's strength equals the other's screw number. In our construction, the Bessel superpositions (3.2) possess an infinite number of dislocation rings in addition to the degenerate structure that unfolds to our knot, so the straight threading dislocation, regarded as a loop closing at infinity, must have an infinite screw number.

Each wavefront $\chi=$ const. $(\bmod 2 \pi)$ is a (possibly infinite) Seifert surface (Adams 1994), smoothly connected at the knotted and helical dislocations with its counterpart $\chi+\pi$. 


\section{Appendix C. Perturbed dislocation stars are convected}

After perturbation, $L_{n}$ splits into a dislocation consisting of $n$ strands, each with strength 1 . If $m$ and $n$ are coprime, then the strands must form a single dislocation loop, winding $n$ times round the original $z$-axis. Here we show that each strand must turn $m$ times around the others before closing. Consider a section of the knot, labelled by its azimuth $\phi$. The section will contain $n$ phase stars. Far from the strands, the pattern of phase contours is unchanged by the perturbation, and so must turn $m / n$ times during a circuit of $A_{m}$, that is, as $\phi$ increases by $2 \pi$. To show that the pattern of stars must also rotate $m / n$ times, we must look more closely.

Each pair of stars will be separated by one or more distinct phase contours, each of which arrives from afar and hits one of the phase saddles between the stars, as in parts $(d)-(f)$ of figure 2. Consider the phase $\chi(\phi)$ corresponding to one of these saddles, as $\phi$ increases by $2 \pi$. If $\chi(\phi)$ is single valued, that is, if this phase does not change by a multiple of $2 \pi$, then the phase contours issuing from this saddle must also turn $m / n$ times, in synchrony with the phase contours far away. Therefore, the stars separated by the phase contours issuing from the saddle must rotate too. An appropriate image is of a 'saddle paddle', convecting the phase stars. The result of this process is that the strands must twist, as claimed.

To show the single valuedness of $\chi(\phi)$ during the circuit, consider the model (cf. $(2.2),(2.6),(2.8)$ )

$$
\psi(\boldsymbol{r})=\exp (\mathrm{i} m \phi)\left[K_{+}(x+\mathrm{i} y)^{n}+K_{-}(x-\mathrm{i} y)^{n}\right]+\varepsilon \psi_{p}(\boldsymbol{r}),
$$

where $x$ and $y$ are local coordinates centred on the place where $L_{n}$ pierces the $\phi$ section. At the saddles, the current $\boldsymbol{j}=\operatorname{Im} \psi^{*} \nabla \psi=0$ but $\psi \neq 0$. To lowest order in $\varepsilon$, there is a saddle at $x=y=0$ (if $n>2$, this is a degenerate saddle, as in figure $2 e$, corresponding to a partial unfolding, but this does not affect the argument). The phase of this saddle is

$$
\chi(\phi)=\arg \left(\psi_{p}(x=0, y=0, \phi)\right),
$$

and this is single valued as $\phi$ changes by $2 \pi$, from our assumption that $\psi_{p}$ possesses no dislocations threading $L_{n}$.

The stipulation that $\psi_{p}$ possesses no dislocations threading $L_{n}$, so that the phase of $\psi_{p}$ is single valued on $L_{n}$, is essential. Without it, we would, for example, be free to choose $\psi_{p}$ proportional to $\exp (\mathrm{i} m \phi)$, and then, simply by reversing the tuning of parameters $a_{l}$ that created the strength $n$ dislocation $L_{n}$, we could unfold it into $n$ separate unlinked loops, that is, the convection necessary for the formation of a knot would not occur.

\section{Appendix D. No higher-order paraxial dislocation loops}

Let the paraxial direction be $z$. Any loop, of whatever shape, must have at least two points where its direction is perpendicular to the $z$-axis. This is obvious geometrically, and also follows from the fact that the tangent vector $\boldsymbol{t}$ to any closed loop varies periodically round the loop, with mean zero, so that any component of $\boldsymbol{t}$ must pass through zero at least twice. Therefore, the absence of paraxial loops with strength $|n|>1$ follows, if it can be shown that the paraxial wave equation

$$
2 \mathrm{i} \partial_{z} \psi+\partial_{x}^{2} \psi+\partial_{y}^{2} \psi=0
$$

prohibits a strength $|n|>1$ dislocation perpendicular to the $z$-axis.

Proc. R. Soc. Lond. A (2001) 
To establish this prohibition, let the dislocation pass through the origin in the $y$-direction. Then the $y$ variation (along the dislocation) must be slower than the $x$ and $z$ variations, so the term $\partial_{y}^{2} \psi$ in (D 1) must be dominated by the other two. Thus, in the $x, z$-plane, $\psi$ must satisfy

$$
2 \mathrm{i} \partial_{z} \psi+\partial_{x}^{2} \psi=0
$$

It is immediately clear that there is no solution proportional to $\left[\sqrt{ }\left(x^{2}+z^{2}\right)\right]^{n}$, but it is instructive to examine in more detail the requirements for a dislocation of strength $n$. Not only must all derivatives $\partial_{x}^{j} \partial_{z}^{p-j} \psi$ of order $p<|n|$ vanish, but all derivatives of $\partial_{x}^{j} \partial_{z}^{n-j} \psi$ of order $n$ must not vanish (cf. (2.5)). However, repeated differentiation of (D 2) shows that this is impossible: if the derivatives less than $n$ vanish, then at least one higher derivative with respect to $x$ must vanish too, spoiling the construction.

The prohibition operates in subtle ways. For example, in (3.1), the innocent replacement of $\sqrt{ }\left(1-b^{2}\right)$ by its paraxial equivalent $1-\frac{1}{2} b^{2}$ results in equations similar to (3.4), which can be solved for the coefficients in the (now paraxial) superposition with $\psi=\partial_{R} \psi=\partial_{z} \psi=0$ at $R=R^{*}$. However, this solution also has $\partial_{R}^{2} \psi=0$ at $R=R^{*}$, so what has been created is a degenerate singular ring with strength unity, rather than the desired strength 2 dislocation ring; another singularity, whose strength is opposite to each of the ones seeking to combine, has arrived, producing a cancellation. Such unwanted guests are paraxially unavoidable. Reflecting this, the numerical solution of (3.4) gets progressively more difficult as the transverse wave numbers $b$ in (3.1) get smaller, that is, as the limit of paraxiality is approached.

\section{References}

Adams, C. C. 1994 The knot book. San Francisco, CA: Freeman.

Allen, L., Beijersbergen, M. W., Spreeuw, R. J. C. \& Woerdman, J. P. 1992 Orbital angular momentum of light and the transformation of Laguerre-Gaussian laser modes. Phys. Rev. A 45, 8185-8189.

Berry, M. V. 1981 Singularities in waves and rays. In Les Houches Lecture Series Session 35 (ed. R. Balian, M. Kléman \& J.-P. Poirier), pp. 453-543. Amsterdam: North-Holland.

Berry, M. V. 1994 Evanescent and real waves in quantum billiards and Gaussian beams. J. Phys. A 27, L391-L398.

Berry, M. V. 1998 Wave dislocations in nonparaxial Gaussian beams. J. Mod. Opt. 45, 18451858.

Berry, M. V. 2001 Knotted zeros in the quantum states of hydrogen. Found. Phys. 31, 659-667.

Berry, M. V. \& Dennis, M. R. 2000 Phase singularities in isotropic random waves. Proc. R. Soc. Lond. A 456, 2059-2079.

Cromwell, P., Beltrami, E. \& Rampicini, M. 1998 The Borromean rings. Math. Intelligencer 20, 53-62.

Durnin, J. 1987 Exact solutions for nondiffracting beams. I. The scalar theory. J. Opt. Soc. Am. A 4, 651-654.

Durnin, J., Miceli Jr, J. J. \& Eberly, J. H. 1987 Diffraction-free beams. Phys. Rev. Lett. 58, 1499-1501.

Karman, G. P., Beijersbergen, M. W., van Duijl, A. \& Woerdman, J. P. 1997 Creation and annihilation of phase singularities in a focal field. Opt. Lett. 22, 1503-1505.

Karman, G. P., Beijersbergen, M. W., van Duijl, A., Bouwmeester, D. \& Woerdman, J. P. 1998 Airy pattern reorganization and sub-wavelength structure in a focus. J. Opt. Soc. Am. A 15, 884-899.

Proc. R. Soc. Lond. A (2001) 
Kelvin, Lord 1867 On vortex atoms. Phil. Mag. 34, 15-24.

Kelvin, Lord 1869 On vortex motion. Trans. R. Soc. Edinb. 25, 217-260.

Moffatt, H. K. 1969 The degree of knottedness of tangled vortex lines. J. Fluid Mech. 35, 117-129.

Moffatt, H. K. \& Ricca, R. L. 1992 Helicity and the Calugareanu invariant. Proc. R. Soc. Lond. A 439, 411-429.

Nye, J. F. 1998 Unfolding of higher-order wave dislocations. J. Opt. Soc. Am. A 15, 1132-1138.

Nye, J. F. 1999 Natural focusing and fine structure of light: caustics and wave dislocations. Bristol: Institute of Physics.

Nye, J. F. \& Berry, M. V. 1974 Dislocations in wave trains. Proc. R. Soc. Lond. A 336, 165-90.

Nye, J. F., Hajnal, J. V. \& Hannay, J. H. 1988 Phase saddles and dislocations in two-dimensional waves such as the tides. Proc. R. Soc. Lond. A 417, 7-20.

Soskin, M. S. (ed.) 1997 Singular optics. Washington, DC: Optical Society of America.

Vasnetsov, M. \& Staliunas, K. (eds) 1999 Optical vortices. Commack, NY: Nova Science.

Winfree, A. T. 1987 When time breaks down. Princeton, NJ: Princeton University Press.

Winfree, A. T. \& Strogatz, S. H. 1984 Singular filaments organize chemical waves in three dimensions. Physica D 13, 221-233.

Winfree, A. T., Winfree, E. M. \& Seifert, H. 1985 Organizing centers in a cellular excitable medium. Physica D 17, 109-115. 
